\title{
EL DISCURSO EN EL CONTEXTO DE LA INTERACGIÓN PERSONAL Y CON EL ENTORNO EN LITERATURA
}

\author{
FERNANDO POYATOS \\ Universidad de New Brunswick, Canadá
}

\section{RESUMEN}

El estudio del discurso en diversas disciplinas suele ignorar muchos de los aspectos no verbales de la interacción personal y con el entorno -en cuyo ámbito se desarrolla nuestro hablar (en términos de lo que ocurre dentro y alrededor del discurso mismo)-, para empezar, desdeñando en él lo que no ha ocurrido como algo meramente incidental, contextual o marginal. Este trabajo, además de intentar corregir esa lamentable y empobrecedora realidad, muestra cómo el investigador, en un análisis experimental u observacional del discurso, o como estudioso de la literatura, se beneficiaría enormemente del prácticamente inexhaustible tesoro de material ilustrativo que ofrecen las literaturas creativas de las distintas culturas. Para ello se resumen brevemente una serie de perspectivas metodológicas necesarias en el estudio de las interacciones en general, identificando: los componentes personales y extrapersonales en el desarrollo de un encuentro, tanto cara a cara como con cuanto nos rodea; nuestra percepción sensorial y cómo las asociaciones sinestésicas fisiopsicológicas funcionan también como componentes del encuentro; los elementos sensibles e inteligibles que funcionan independientemente o en complejos dobles o múltiples; los calificadores de las actividades y no-actividades interactivas; y cómo los componentes de la interacción se relacionan con otros precedentes, simultáneos o siguientes, todo lo cual enriquece el estudio del discurso, siempre verbal-no verbal.

PALABRAS CLAVE: discurso, interacción, comunicación no verbal, literatura creativa.

\section{ABSTRACT}

Discourse analysis in different disciplines tends to ignore many of the nonverbal aspects of interaction, both interpersonal and with the environment, the realm in which we develop our speech (in terms of what takes place within and around it), neglecting, to begin with, what has not happened within speech as something merely incidental, contextual or marginal. The present study, besides trying to correct this unfortunate and impoverishing reality, shows how the researcher, in an experimental or observational analysis of speech, or as a student of literature, would greatly benefit from the practically inexhaustible wealth of illustrations offered by the creative literatures of the different cultures. To that purpose, 
it briefly outlines a series of methodological perspectives needed for the study of interactions in general, identifying: the personal and extrapersonal components in the development of an encounter, whether face-to-face or between us and whatever surrounds us; our sensory perception and how physiopsychological synesthesial associations operate as well as components of the encounter; the sensible and intelligible elements operating independently or in double or multiple clusters; the qualifiers of interactive activities and nonactivities; and how interaction components relate to preceding, simultaneous or succeeding components, all of which enrich the study of discourse, which is always verbal and nonverbal.

KEYWORDS: interaction, conversation, nonverbal communication, creative literature.

\section{LA LiTERATURA COMO FUENTE PARA EL ESTUDIO DE LA COMUNICACIÓN NO VERBAL EN EL DISCURSO}

Cuando en los últimos años sesenta me esforzaba por desarrollar, siempre interdisciplinarmente, los estudios de comunicación no verbal (como cursos en los departamentos de Antropología, Sociología y Psicología de mi Universidad y como simposios en numerosos congresos en estas y otras disciplinas, como lingüística o semiótica), unos alentadores comentarios sobre un artículo mío en Revista de Occidente (Poyatos, 1972a) de su entonces secretario, el filósofo Paulino Garagorri, y de Camilo José Cela -en cuya revista Papeles de Son Armadans publiqué más tarde, más dos extensos artículos que me pidió años después para su segunda revista, El Extramundi y los Papeles de Iria Flavia (Poyatos, 1997, 2001)-, el premio de la Asociación Canadiense de Hispanistas a mi trabajo en un volumen sobre teoría de la novela (Poyatos, 1976), y el que siguió en Semiotica, la revista del gran Thomas Sebeok (Poyatos, 1977), que decidió reeditar en un volumen de "artículos pioneros" de su serie Approaches to Semiotics (Poyatos, 1981), me confirmaban las ricas perspectivas que las literaturas nacionales ofrecían para el estudio de la comunicación no verbal como parte y a la vez medio envolvente del discurso en cada lengua y cultura.

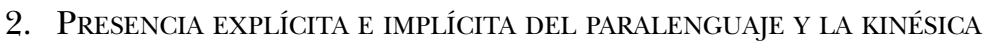

Para concentrarnos en el tema aquí propuesto -la interacción interpersonal y con el entorno como ámbito en que se desarrolla el discurso oral-, excluyamos la más obvia presencia explícita de sus dos componentes no verbales, paralenguaje y kinésica ${ }^{1}$ (presentes de alguna forma en los ejem-

${ }^{1}$ Ambos campos están de sobra definidos y diferenciados: para el paralenguaje existe una única y extensa monografía (Poyatos, 1993), que hoy hubiera ilustrado mucho más pro- 
plos que siguen), e indiquemos, al menos sumariamente, su presencia implícita, que tanto se descuida en trabajos a menudo un tanto precipitados ${ }^{2}$.

- $\quad$ El paralenguaje implícito en el texto (dependiendo de lo alejado que el lector pueda estar cultural o históricamente, o ambos) lo percibimos:

\section{- por la conducta kinésica del personaje:}

-¿Si me cayera ahí...? / -No lo contabas. / -¡Qué miedo, chico! / Hizo un escalofrío con los hombros (Sánchez Ferlosio, J, 199);

- cuando, además, se indica el significado de esa conducta kinésica:

-Quita, quita -clamaba la señora con expresión de asco- ¿Me tomas por esas...?] (Galdós, D, VIII);

- por la conocida correlación proxémica-voz:

Puis elle se leva, s'approcha de l'Allemand pour lui parler a l'oreille, et lui dit: -Dieu merci! le voilà qui va s'endormir (Balzac, $C P, 248$ );

- por la conocida correlación entre otras reacciones somáticas y el paralenguaje (ej., sonrojo, llanto):

Ella [Mary, que estaba llorando antes, a Walter, íntimo amigo de su marido recién muerto] se le abrazó y le besó en la mejilla [...] / "Vamos, vamos," dijo él, con un intenso sonrojo y tratando de abrazarla y sostenerla sin tocarla muy de cerca. "Vamos, vamos," dijo otra vez [...] [y cuando la familia le da las gracias por haberlos visitado] [...] Meneó la cabeza. "Nada, no es nada," dijo. (Agee, $D F, \mathrm{X})^{3}$;

- por el contexto situacional externo descrito, quizá específicamente cultural:

"Pero... tienes que comer, Jay. Aún hará fresco unas cuantas horas" [cuando se levanta en mitad de la noche]. Hablaba como en una iglesia o en una biblioteca, por los niños, que dormían, inconscientemente y por la hora de la noche que era (Agee, $D F$, II $)^{4}$;

fusamente con ejemplos literarios; en cuanto a la kinésica, Emilio Lorenzo, en un artículo para $A B C$ (1999), justificó detalladamente la legitimidad de mi grafía $k$, y yo expliqué repetidamente que (aparte de la posible grafía qu) el usar $c$ (cinésica) obligaría al estudioso a referirse a ciertas propuestas unidades kinésicas como cine (kine), cinema (kinema), etc..

${ }^{2}$ Debo advertir (con gratitud para quienes se interesen en mis trabajos) que la mayoría de los temas en mi trilogía en español (Poyatos, 1994a, b, c) están bastante ampliados en otra posterior (Poyatos, 2002a, b, c) -en inglés y con muchísimas más ilustraciones literariasy en otros trabajos en ambos idiomas, que recomiendo a los investigadores para actualizarse en los diversos campos incluidos.

3 «She threw her arms around him and kissed him on the cheek [...] "There now," he said, blushing deeply and trying to embrace and to sustain her without touching her too closely. "There now," he said again [...] [...] He shook his head. "Nothing, Nothing," he said".

4 «"Well, you got to eat, Jay. It'll still be chilly for hours." She spoke as if in a church or library, because of the sleeping children, unconsciously, because of the time of night». 
- por la personalidad del personaje, su estado emocional, etc.:

[Hetty, en su celda, antes de ser ahorcada] “¿No vas a dejarme,

Dinah? ¿Te quedarás cerca de mí?” (Eliot, $A B, \mathrm{XLV})^{5}$;

- por los rasgos paralingüísticos que al lector nativo, o muy familiarizado con el idioma, le evocan la lectura de otros verbales o no verbales:

EMILY. ¿Quieres decir la casa y todo? / GEORGE. Síii (Wilder, OT, I) ${ }^{6}$.

- La kinésica implícita en el texto (con los mismos posibles problemas) la percibimos:

- por las palabras del personaje:

- ¿A mí? Por aquí me entra y por aquí me sale (Sánchez Ferlosio, $214)^{7}$

- por su conducta paralingüistica cuando se refleja verbalmente y con la puntuación:

-¡Mira; si no te callas...! -amagaba Mauricio (Sánchez Ferlosio, 47);

- por otra conducta kinésica, como la expresión facial que en este ejemplo imaginamos por la manual:

Ántonia trató de explicar. "Esto [lo que la señora Shimerda había dado a la señora Burden] muy bueno, señora Burden -se cogía las manos como si no pudiera expresar cómo de bueno-" [...] ¡oh, tan bueno! (Cather, $M A, 78)^{8}$;

- por reacciones químicas, como las lágrimas:

Sancho $[\ldots]$, compungiéndose de manera que le vinieron las lágrimas a los ojos $[\ldots]$ con voz dolorida y enferma le dijo [...] (Cervantes, $Q, 2, \mathrm{XXVIII)}$;

- por la conocida correlación entre la conducta kinésica y ciertas reacciones dérmicas:

"[...] tiene que tener mucho cuidado al bajar esos escalones. Son terriblemente escurridizos / Illidge [cojo] se sonrojó. “¡No, qué va!,” murmuró y enrojeció aún más intensamente [...] dándose cuenta de la imbecilidad que había dicho (Huxley, PCP, IV) ${ }^{9}$;

- el buen lector, por la personalidad del personaje, si su creador nos la ha ofrecido inicialmente y ha mantenido consistentemente su configuración kinésica.

5 «"You won't leave me, Dinah? You'll keep close to me?”».

6 «EMILY. You mean the house and everything? / GEORGE. Yeah».

7 Dando en los años sesenta un curso de novela española a angloparlantes, tuve que aclarar, ante sus sonrisas, que se trataba de la expresión inglesa "It goes in one ear and out the other", que no conlleva el gesto.

8 «Ántonia undertook to explain. "This very good, Mrs. Burden -she clasped her hands as if she could not express how good-" [...] oh, so good!».

9 « "[...] you must really be careful coming down those stairs. They are terribly slippery." / Illidge blushed. "Not at all," he muttered and blushed still more deeply [...] as he realized the imbecility of what he had said». 


\section{EL ENFOQUE REALISTA DE LA INTERACCIÓN Y EL DISCURSO}

Cuando hace unos cuarenta años empecé a reconocer la complejidad de la interacción interpersonal y con el entorno, veía que la psicología social y otras disciplinas tendían a descuidar muchas ocurrencias como meramente incidentales, contextuales o marginales, identificando a lo sumo una serie de actos interactivos en una secuencia causa-efecto e ignorando lo que no ocurría, aún más lo que hubiera o no hubiera ocurrido (de haber tenido lugar una actividad, como un gesto o una palabra, o una no-actividad, como el vestido o el estatus social), y no digamos lo que no habia ocurrido aún o ni siquiera iba a ocurrir. Así que, considerando la presencia del discurso (o sus efectos implícitos) en la interacción personal o con el entorno, empecé a concebir la interacción como:

El intercambio consciente o inconsciente de signos comportamentales o no comportamentales, sensibles o inteligibles dentro de todo un arsenal de sistemas somáticos y extrasomáticos (independiente de que sean actividades o no-actividades o de que hayan ocurrido o no vayan a ocurrir en absoluto) y del resto de los sistemas culturales circundantes, puesto que todos ellos actúan como componentes emisores de signos (y como posibles generadores de otras emisiones) que determinan las características específicas de ese intercambio.

Simultáneamente iba descubriendo una fuente de datos prácticamente inexhaustiva dentro de la literatura creativa, incluso para la investigación interdisciplinar dentro de las ciencias sociales (Poyatos, 2002d, 2002e, 2015). Aquí resumiré una serie de perspectivas metodológicas, concretamente para el estudio de: los componentes personales y extrapersonales de la interacción cara a cara y con el entorno; su percepción sensorial y las asociaciones sinestésicas fisiopsicológicas; cómo los componentes sensibles o inteligibles pueden funcionar independientemente o en construcciones dobles o múltiples; los calificadores de las actividades y no-actividades interactivas; y cómo todo componente de una interacción interpersonal o con el entorno puede relacionarse significativamente con otros componentes verbales o no verbales, precedentes, simultáneos o siguientes, lo cual ofrece también amplias perspectivas de investigación y docencia, incluyendo el estudio de la literatura. Omito el tema, por otra parte insoslayable, de la estructura de la conversación y cualquier comentario introductorio sobre el discurso verbal-no verbal y, dentro de él, del paralenguaje y la kinésica en el contexto literario, que ya he tratado en profundidad, limitándome aquí a adherirme muy brevemente al esquema adjunto, "La interacción personal y con el entorno como comunicación no verbal en literatura", sin salirnos de la literatura como fuente de datos y confiando 


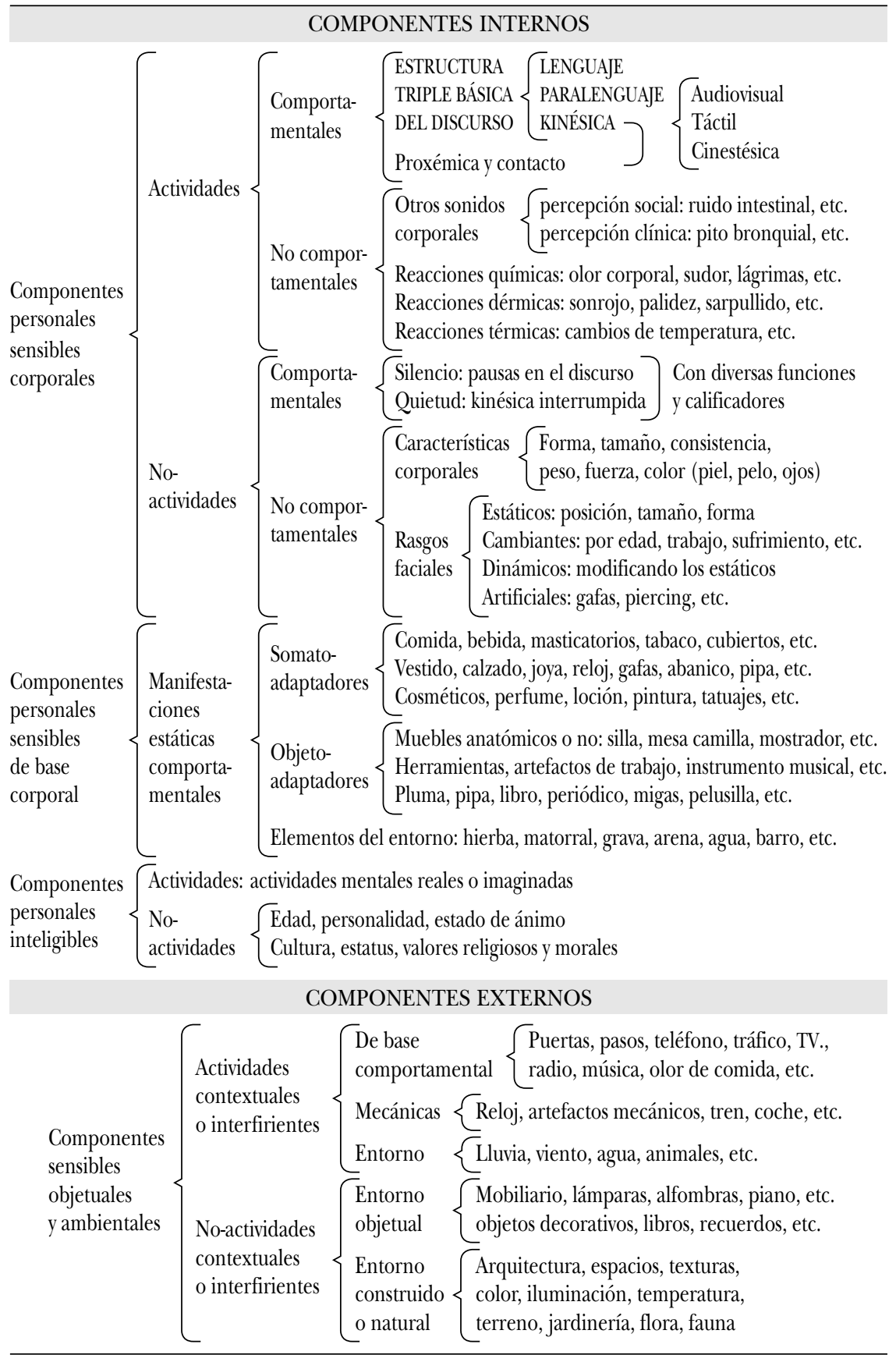

La interacción personal y con el entorno como comunicación no verbal en literatura 
en que sugerirá diversas perspectivas de investigación y enseñanza, según la disciplina o especialidad de cada uno. Los ejemplos literarios, tomados de entre los miles contenidos organizadamente en un voluminoso Literary Thesaurus of Nonverbal Communication for Interdisciplinary Research (aún inédito), los traduzco del inglés, poniendo el original a pie de página.

\section{LOS COMPONENTES DE LA INTERACCIÓN JUNTO CON EL DISCURSO:}

INTERNOS, EXTERNOS, PERSONALES, OBJETUALES Y AMBIENTALES, Y LA LITERATURA CREATIVA COMO FUENTE DE ESTUDIOS INTERDISCIPLINARES

En uno de los tres cursos sobre comunicación no verbal que he mencionado, el que diseñé para el Departamento de Psicología, las primeras ocho o diez clases las dedicaba a analizar lo que llamábamos los niveles profundos de la interacción, empezando por afirmar que no podemos tener una imagen completa de una situación interactiva dada y analizar lo que pasa exactamente -para luego determinar cómo y por qué las cosas pasan como pasan- sin antes identificar todos los componentes de esa situación, en lugar de decidir arbitrariamente que uno o más no cumplen una función interactiva y que, por tanto, no merecen nuestra atención. Porque si realmente los identificáramos todos -como en el esquema adjunto-, veríamos que lo que puede parecer un vacio semiótico está bien lejos de serlo, pues hay signos no verbales (ej., la mirada distraída de uno de los cointeractores mientras nos dice algo) con funciones bien concretas, sin los cuales no percibimos una serie de interrelaciones -entre elementos verbales y no verbales (aun dentro del discurso) y entre los no verbales- e importantes complejos de signos y mensajes que a su vez suscitan otras actividades o no-actividades, mostrando las muchas perspectivas interdisciplinares que pueden desarrollarse con este modelo. Por ejemplo:

- el desarrollo personal de la estructura lenguaje-paralenguaje-kinésica (y no meramente el lenguaje verbal, ni necesariamente aparte de otros sistemas, como la proxémica), puesto que los tres repertorios (el verbal, el paralingüístico y el kinésico) tienen un claro desarrollo morfológico y funcional en cada uno de nosotros (y cualquier desequilibrio puede delatar diversas anomalías en ese desarrollo);

- un estudio sociopsicológico transcultural de la conceptualización y funciones de los silencios interactivos como pausas en el discurso y, concretamente, en la conversación;

- las muchas implicaciones sociopsicológicas en el uso de los llamados somatoadaptadores (ej., comiendo, bebiendo, masticando chicle, 
cómo se lleva la ropa, el uso del mobiliario anatómico) y objetoadaptadores (ej., la mesa camilla, una herramienta);

- el efecto en una interacción personal, incluso en el discurso mismo, de los componentes inteligibles (rango social, autoridad, religión, etc.) en un encuentro, según edad, personalidad y cultura;

- las relaciones profundas entre el entorno construido (trazado urbano, espacios y volúmenes arquitectónicos) y la interacción interpersonal y la de la persona con el entorno.

La primera diferenciación básica es entre dos categorías fundamentales de componentes interactivos: internos (elementos activos o pasivos, signos que los participantes pueden intercambiar entre ellos, o con una relación directa en el intercambio, como el lenguaje verbal, el perfume o el estatus social); y externos (actividades comportamentales y no-comportamentales y elementos estáticos, que no pueden ser parte del mutuo intercambio personal, pero que ocurren en la periferia del encuentro, aunque no necesariamente disociados del discurso).

\section{COMPONENTES INTERNOS}

A. COMPONENTES PERSONALES SENSIBLES CORPORALES, producidos por actividades o no-actividades somáticas, y comprenden tres grupos:

1. Actividades personales sensibles comportamentales

- La estructura triple básica del discurso: lenguaje verbal-paralenguajekinésica, centro de nuestra comunicación verbal-no verbal (por eso aparece en MAYÚSCULAS en el esquema).

- El PARALENGUAJE (Poyatos, 1993), modificaciones de la voz por: cualidades básicas, como registros; tipos de voz, como susurrante o atiplada; y emisiones independientes o cuasipalabras, como clareos de garganta, gemidos, silencios momentáneos. Aparte de las descripciones verbales, el paralenguaje está, más aún, implícito en el texto literario, como ya se ha ilustrado:

«Non fables muy apriesa nin otrosi muy paso,/ [...] quien fabla muy paso, enójase quien le atiende» (Ruiz, $L B A, 550 \mathrm{a}, \mathrm{b}, 551 \mathrm{~b}$ ), «afinándola [la vihuela] lo mejor que pudo, escupió y remondóse el pecho, y luego con una voz ronquilla, aunque entonada, cantó» [...] (Cervantes, $Q$, 2, XLVI), «-¡Psche! Según como lo quiera mirar» (Cela, VA, IV).

- La KINÉSICA, movimientos y posiciones de ojos, cara, tronco y extremidades, de percepción visual, auditiva, táctil y cinestési- 
ca; está siempre en el texto, explícitamente descrita o, como el paralenguaje, implícita (;con el problema intercultural de la traducción!) y percibida, y aun sin verla, se asocia a otras conductas:

«Apretó las manos [durante su discurso en un soliloquio) y sintió la dureza de las pequeñas monedas que tenía cogidas» (Woolf, $Y$, "Tiempo presente") ${ }^{10}$.

- La proxémica, o sea, la conceptualización y estructuración comportamental, consciente o inconsciente, de nuestros espacios interpersonales, incluyendo el contacto, y con relación al entorno:

La separé de mí [a Laura] sorprendida [...] "No te enfades conmigo, Mariam," dijo, interpretando mal mi silencio. / Yo respondí solo atrayéndola hacia mí de nuevo, por miedo a llorar si hablaba (Collins, WW, 186-187) ${ }^{11}$.

- $\quad$ Sonidos corporales más allá de lenguaje y paralenguaje (como actividades intencionadamente comportamentales o incontrolables) de significado social o clínico, como el ruido intestinal (a veces embarazoso) o el pito bronquial provocado por asma, alergia a un gato o ansiedad social (ambos componentes de la interacción).

\section{Actividades personales sensibles no-comportamentales}

Son reacciones incontrolables (a menudo reflejos) de percepción olfativa, visual, dérmica o gustativa, a veces con funciones interactivas portadoras de mensajes con valor social o clínico.

- $\quad$ Reacciones químicas, como sudor fisiológico o emocional (ej., el sudor palmar durante una entrevista o interrogatorio), lágrimas silenciosas o en el discurso (ej., con señales visibles de autocontrol), el olor de fallo hepático, de congelamiento, o el de una crisis esquizofrénica, percibido por algunos sanitarios, incluso el de muerte inminente):

a Cardenio se le mudó la color del rostro, y comenzó a trasudar [...] y estarse quedo, mirando de hito en hito a la labradora, imaginando quién era ella [Dorotea] (Cervantes, $Q, 1, \mathrm{XXVIII)}$;

por supuesto, son susceptibles de una íntima interacción a través del tiempo y de interpretaciones negativas con valor interactivo (por racismo, prejuicios de clase, etc.).

10 «She clenched her hands and felt the hard little coins she was holding».

11 «I held her away from me in astonishment [...] "Don't be angry with me, Mariam," she said, mistaking my silence. / I only answered by drawing her close to me again. I was afraid of crying if I spoke». 
- Reacciones dérmicas, como sonrojo emocional, palidez y carne de gallina:

Con las primeras palabras de ella el color se le había subido a la frente a [Owen]; pero al seguir vio [ella] que se le serenaba la cara y la sangre remitía (Wharton, $R$, XVIII) ${ }^{12}$.

- Reacciones térmicas, como subidas y descensos de la temperatura corporal, generalmente junto con otra actividad fisiológica:

Oí que su respiración [de Laura] se aceleraba -sentí su mano enfriarse (Collins, WW, 191) ${ }^{13}$.

3. No-actividades personales sensibles comportamentales o no-comportamentales Pueden ser tan importantes como lo que expresamos verbalmente o por gestos y posturas, por sí solos o combinados con otros componentes, y hay tres tipos.

- $\quad$ Silencio y quietud, con diversas funciones calificados por su intensidad y su duración, que influyen, intencionadamente o no, en la interacción (Poyatos, 1998; 2002c: cap. 7; 2013):

Se hablaban en voz baja con tímida mesura, y en los momentos de silencio oíase el péndulo de un reloj (Valle Inclán, SP, 33).

- Características corporales estáticas de forma, tamaño, consistencia, peso, fuerza (percibida táctilmente y por el sentido de la cinestesia $^{14}$, conscientemente o no), color de piel, pelo y ojos, y los rasgos faciales, los cuales filtran nuestro discurso y pueden ejercer importantes funciones interactivas que pueden afectarlo:

«[durante su diálogo] Todavía le retenía él la mano [...] En la cálida presión se notaba su fuerza, y esto la confortó» $(\text { Grey, } L T, \mathrm{VI})^{15}$, «Su ligera corpulencia [Roy Kear] aumentaba más su dignidad. Daba peso a sus observaciones» (Maugham, CA, II $)^{16}$.

- La cara hablando o en silencio, principal canal de comunicación, muestra cuatro tipos de rasgos faciales (Poyatos, 2002a: 63-74):

\footnotetext{
12 «At her first words the colour had rushed to his forehead; but as she continued she saw his face compose itself and his blood subside».

13 «I heard her breath quickening - I felt her hand growing cold».

${ }^{14}$ A través de articulaciones, músculos, tendones y nervios percibimos la posición del cuerpo y sus miembros en el espacio y en cualquier contacto (al volante, en un abrazo, caminando, etc.).

${ }_{15} \ll$ He still held her hand [...] A sense of his strength came with the warm pressure, and comforted her».

${ }^{16}$ «His slight corpulence [Roy Kear] only added to his dignity. It gave weight to his observations».
} 
- permanentes, es decir, posición, tamaño y forma de cejas, párpados y pestañas, nariz, mejillas, boca, frente, barbilla y mandíbula, a las que puede añadirse a largo plazo un tipo de peinado, una barba, etc.:

con pequeñas y gorditas mejillas de ardilla y una boca perpetuamente dispuesta para enjuiciar con desdeño [...] engreído e insufrible en todos los sentidos (Doctorow, WF, XIII) ${ }^{17}$;

- cambiantes (por tiempo, edad, trabajo, sufrimiento, hábitos), como arrugas, pliegues, manchas, etc.:

Se le marcaban en el cuello los músculos adquiridos por el hábito de toda la vida de apretar la mandíbula y adelantarla [también hablando] (MacLennan, TS, 1, XI) ${ }^{18}$;

- dinámicos, positivos o negativos como parte del discurso al modificar su estado estático (ej., en un retrato), crucial en un primer encuentro (ej., una entrevista de trabajo):

Y hablando [Jacinta], sus atractivos eran mayores que cuando estaba callada, a causa de la movilidad de su rostro y de la expresión variadísima que sabía poner en él (Galdós, FJ, 1, IV, II);

- artificiales, realzando o debilitando los rasgos naturales:

Llevando la marca de bermellón donde empieza la raya central del pelo, como debe hacer una mujer, ganaría libertad, libertad para vivir a su manera [afectando también a su manera de hablar (Bhattacharya, HWRT, XXVI) ${ }^{19}$.

B. COMPONENTES PERSONALES SENSIBLES DE BASE CORPORAL, que operan en la interacción como elementos estáticos o manifestaciones comportamentales cuando son parte (o, más bien, condicionantes) de actividades kinésicas, y comunican por sus propias características y por las conductas asociadas a ellos.

1. Somatoadaptadores, objetos y substancias más íntimamente en contacto con el cuerpo, que pueden influir consciente o inconscientemente incluso en nuestro discurso.

17 «with fat little squirrel cheeks and a mouth perpetually primed in contemptuous judgement $[\ldots]$ in all ways smug and insufferable».

${ }_{18}$ «His neck was ridged with muscles acquired from a life-long habit of stiffening his jaw and pushing it forward».

19 «Wearing the vermilion mark of marriage at the central parting of her hair, as a woman must, she would gain freedom, freedom to live her own way». 
- Comida, bebida, tabaco, masticatorios y objetos relacionados con ellos (cubiertos, palillo de dientes, pipa, etc.), a través de los cuales (a veces hablando) manifestamos estatus social, estado de ánimo, etc., en diversas circunstancias:

«Los peregrinos comenzaron a comer con grandísimo gusto y muy de espacio, saboreándose con cada bocado, que le tomaban con la punta del cuchillo, y muy poquito de cada cosa, y luego [...] levantaron los brazos y las botas en el aire; puestas las bocas en su boca, clavados los ojos en el cielo [...] meneando las cabezas a un lado y a otro, señales que acreditaban el gusto que recebían» (Cervantes, $Q, 2$, LIV), «[el lascivo mercader] Abrió la boca en una especia de mueca, los dientes rojos por el jugo de la hoja de betel» ${ }^{20}$ (Bhattacharya, HWRT, II), «[Waythorn] abrió su petaca y se la alargó a su visitante [el primer marido de su mujer], y el que Haskett lo aceptara pareció marcar una nueva fase en su relación [...] Waythorn invitó a su visitante a acercar una silla al fuego [...] Los dos se envolvieron en la intimidad [compartida] del humo de sus puros» (Wharton, $O T, \mathrm{~V})^{21}$.

- Ropa, calzado y accesorios, que históricamente han determinado ciertas conductas y comunicado preferencias estéticas, estado de ánimo, intencionadas funciones sociales, descuido patológico en el aseo personal, etc., incluso produciendo sonidos realmente interactivos que hoy raramente se oyen:

«El frufrú de su bonita falda era como música para él» (Dreiser, SC, XXI) ${ }^{22}$, «Don Basilio solía llevar en la boca un palillo de dientes, y tomándolo entre los dedos lo mostraba, accionando con él, como si formara parte del argumento» (Galdós, FJ, 3, III).

- Perfume, cosméticos, pintura, tatuajes, "piercings" y otras marcas corporales, siempre componentes de la interacción:

Solo alguien observador podría haber notado rayas de esfuerzo y agotamiento en su cara que los cosméticos y la fuerza de voluntad no habían podido ocultar del todo (Hailey, $H$, "Miércoles") ${ }^{23}$.

2. Objetoadaptadores, que incluso podemos manipular como conductas kinésicas para afectar a nuestra interacción.

20 «His mouth had opened in a kind of grin, the teeth red with the juice of betel leaf».

${ }^{21}$ «He [Waythorn] opened his cigar case and held it out to his visitor, and Haskett's acceptance seemed to mark a fresh stage in their intercourse. The spring evening was chilly, and Waythorn invited his guest to draw up a chair to the fire [...] The two were enclosed in the intimacy of their blended cigar-smoke».

22 «The rustle of her pretty skirt was like music to him».

23 «Only the observant might have noticed lines of strain and weariness in her face which cosmetics and an effort of will power had not obscured entirely». 
- Mobiliario anatómico o no, al que asociamos nuestra conducta, incluyendo el discurso:

se sentó $[\ldots]$ a horcajadas sobre una silla de tijera puesta del revés, los brazos cruzados sobre el indeciso espaldar (Caballero Bonald, DDS, 2, XII).

- $\quad$ Herramientas, artefactos de trabajo, instrumentos musicales, etc., cuyo manejo puede delatar ciertas actitudes o estados de la persona y hasta el efecto duradero de algo ocurrido antes:

[Tom] se escupió en las manos. El pico se alzó en el aire y cayó fulminante. Tom gruñó suavemente. El pico subía y caía, y el gruñido [paralenguaje] salía en el momento en que se hundía en la tierra [...] (unf) [...] "Tu y el pico (unf) trabajando juntos (unf)" (Steinbeck, GW, XXII) ${ }^{24}$.

¡Vivamos la experiencia de Tom no leyendo esta escena deprisa!

- $\quad$ Objetos que manipulamos como conductas kinésicas que revelan actitudes y estados que pueden afectar a los pensamientos y conductas de nuestros cointeractores, como la pluma con que escribimos, la pelusilla (a veces inexistente) que nos sacudimos de la ropa, la miga de pan con que jugueteamos en la mesa, etc., incluso delatar el efecto duradero de algo ocurrido antes:

«Antonio [delante del ayudante del jefe de personal] daba vueltas al casco» (López Salinas, $M, 77$ ), «El raspear de la pluma [con elocuencia no verbal cuasiléxica] de Eleanor le irritaba» (Woolf, $Y$, “1880") ${ }^{25}$.

3. Elementos del entorno, como agua, barro, arena, hierba, etc., que suscitan ciertas posturas o movimientos que, a su vez, afectan al modo de hablar:

Temple [andando por la arena] haciendo esfuerzos y tambaleándose con sus tacones altos (Faulkner, $S, \mathrm{~V})^{26}$.

C. COMPONENTES PERSONALES INTELIGIBLES, que los investigadores suelen desdeñar, aunque funcionan en la interacción sin interrupción (como a través de las conductas y no-conductas que percibimos) o intermitentemente.

1. Las actividades mentales y la categoría siguiente, no-actividades (reales o no), constituyen el ámbito más profundo de una interacción interpersonal o con el entorno, a veces solo imaginada, pero aun así decisiva en

24 «[Tom] spat on his hands. The pick arose into the air and flashed down. Tom grunted softly. The pick arose and fell, and the grunt came at the moment it sank into the ground [...] $(u m p h)[\ldots]$ "You an' the pick (umph) workin' together (umph)"».

25 «The scratching of Eleanor's pen irritated him».

26 «Temple struggling and lurching on her high heels». 
el encuentro. Yo puedo reaccionar verbal o no-verbalmente al darme cuenta de que otra persona (por sus palabras, su paralenguaje, porque me tiene de pie y no me invita a sentarme) me considera inferior, después de cometer un error que reconozco; pero aunque me equivoque en cuanto esa opinión de mí, reacciono así; o sea, algo que no ha ocurrido en absoluto se hace de lo más real en mi mente y condiciona parte de mi interacción o toda ella. Y, por otra parte, la ansiedad, por ejemplo, pudo suscitarla la convicción de mi verdadera inferioridad delante de alguien que, sin embargo, no piensa lo que yo me creo que piensa:

¿Qué estaba pensando? ¿Se había portado [ella] como una idiota? La voz se le entrecortó, la sangre le subió a las mejillas, miró a su plato; y durante los siguientes breves minutos [él] solo obtuvo breves respuestas entre dientes a sus preguntas, solo una risa nerviosa de lo más superficial (Huxley, EG, XXVI ${ }^{27}$.

2. No-actividades, en las que pensamos por las mismas reacciones mentales suscitadas por pensamientos (reales o no) de nuestro cointeractor, y representadas por factores que, quizá equivocadamente, asociamos con esa persona (ej., personalidad, estatus socioeconómico, valores morales), condicionando, nos demos cuenta o no, nuestro comportamiento verbalno verbal (vocabulario, paralenguaje, kinésica, hasta los temas tratados):

Era una de esas sonrisas irritantes que parecen decir: "Amigo mío, ¿qué puede saber usted del sufrimiento?” Había en ella algo condescendiente y superior intolerablemente esnob (West, $D L, \mathrm{XXII})^{28}$.

\section{COMPONENTES EXTERNOS}

D. COMPONENTES SENSIBLES OBJETUALES Y AMBIENTALES, no intercambiados en los encuentros interpersonales, pero con posible valor interactivo muy concreto y como estímulo para esos intercambios de conductas y actitudes, incluyendo el discurso.

1. Actividades contextuales o interfirientes, que ocurren en la periferia del encuentro (y no siempre percibidos por los participantes), suscitando el discurso o restringiéndolo, o únicamente como elementos contextuales con relación a la interacción interpersonal y sin efecto concreto sobre ella.

27 «What was he thinking? Had she made a fool of herself? Her voice faltered, the blood rose to her cheeks, she looked down at her plate; and for the next few minutes he would get nothing but short mumbled answers to his questions, nothing but the most perfunctory of nervous laughs».

28 «It was one of those irritating smiles that seem to say: "My friend, what can you know of suffering?" There was something very patronizing and superior about it and intolerably snobbish». 
- $\quad$ Generadas por nuestra conducta (ej., un portazo que damos, pasos, el tráfico y la gente en la calle, la televisión, olores de comida):

Se oía el rápido y ligero 'clac' de su aguja, el agudo 'pop' [valor paralingüístico cuasiléxico] de los labios de él al echar el humo, el calor, el crepitar en las barras cuando escupía en el fuego (Lawrence, $S L$, III $)^{29}$.

- Generadas por artefactos:

El sonido del Big Ben golpeaba la media entre ellos [Elizabeth and Peter] con extraordinario vigor, como si un muchacho fuerte, indiferente, desconsiderado, estuviera balanceando unas pesas de un lado a otro. / “¡Peter! ¡Peter!” gritó Clarissa, siguiéndolo hasta el rellano. “¡Mi fiesta! ¡Recuerda mi fiesta esta noche!”, gritaba, teniendo que elevar la voz para contrarrestar el fragor del exterior y ahogada por el tráfico y el sonido de las campanadas de todos los relojes (Woolf, $D, 35)^{30}$.

- Generadas por el ambiente natural (incluyendo el mundo animal), pueden actuar contextualmente en el ánimo de los hablantes y afectar a su lenguaje, su paralenguaje y su kinésica:

el canto de un pájaro, el chapoteo de un pato en el estanque, un susurro del viento en el bosque (Styron, CNT, II) ${ }^{31}$.

2. No-actividades contextuales o interfirientes, relacionadas con factores como la edad, la fluidez interactiva de uno, su capacidad de atención, su configuración psicológica, su estatus socioeducacional, o la naturaleza del encuentro.

- $\quad$ no-actividades objetuales del entorno, que suscitan o condicionan, por ejemplo, nuestra primera impresión de un hogar o de la consulta de un médico (mobiliario, alfombras, lámparas, títulos profesionales, fotos, cuadros originales o reproducciones, tipos de libros o de suscripciones, una mesa elegantemente puesta que puede intimidar a algún comensal e influir en su comportamiento verbal-no verbal, etc.):

La habitación se prestaba a la meditación. La lámpara con un velo rojo, los rincones en sombra, los destellos del fuego en las curvas de los grandes y viejos roperos y armarios, le daban un aire de intimidad realzada por

29 «There would be the swift, slight 'cluck' of her needle, the sharp 'pop' of his lips as he let out the smoke, the warmth, the sizzle on the bars as he spat in the fire».

${ }^{30}$ "The sound of Big Ben striking the half-hour struck out between them with extraordinary vigour, as if a young man, strong, indifferent, inconsiderate, were swinging dumb-bells this way and that. / "Peter! Peter!" cried Clarissa, following him out on the landing. "My party! Remember my party tonight!" she cried, having to raise her voice against the roar of the open air, and overwhelmed by the traffic and the sound of all the clocks».

31 «a bird call, the plashing of a duck on the millpond, a whisper of wind in the forest». 
sus cortinas descoloridas, sus alfombras ligeramente raídas y deshilachadas (Wharton, $R$, XIII) ${ }^{32}$.

- el entorno construido puede actuar como elemento contextual o interfiriente e inspirar ánimo, intimidar, relajar, etc., predisponiéndonos, conscientemente o no, hacia nuestros cointeractores, como ocurre con algunos espacios domésticos (cuya influencia en nuestra vida diaria no la tienen en cuenta los arquitectos con menos sensibilidad) o con elementos relacionados ("discreto", "llamativo", "agobiante"), la iluminación (propicia a una interacción íntima verbal-no verbal, o lo opuesto), temperatura (causa de bienestar o incomodidad fisiopsicológicos), música ${ }^{33}$, etc.:

Muchas veces [ella] dejaba que la oscuridad los envolviera, reprimiéndose de encender la lámpara. La oscura y discreta habitación, su propio aislamiento, la música que aún vibraba en sus oídos, los unía (Joyce, $P C)^{34}$;

son elementos que evidentemente afectan a la interacción verbalmente y no verbalmente.

- $\quad$ el entorno natural, como un amplio paisaje que inspira libertad (o agorafobia), sonidos naturales, imágenes y sonidos de animales:

El mundo entero vacío parecía encantado. Susurros de la salvia, la arena filtrándose, ráfagas de viento, la noche, la soledad (Grey, UPT, XIII) ${ }^{35}$.

- una combinación de componentes ambientales y personales puede afectar a nuestro discurso consciente o inconscientemente:

«Dentro estaba oscuro, una prolongada bocanada de aire caliente, cargado de los olores mezclados de flores, perfume, tapicería y gas, la envolvió instantáneamente. Era el inconfundible aroma del teatro [final del siglo XIX, EE.UU.]» (Norris, $P, \mathrm{I})^{36}$, «[Jolyon y su prima Irene] En aquel

32 «The room was propitious to meditation. The red-veiled lamp, the corners of shadow, the splashes of firelight on the curves of old full-bodied wardrobes and cabinets, gave it an air of intimacy increased by its faded hangings, its slightly frayed and threadbare rugs».

${ }^{33}$ En 2005, en la Facultad de Musicología de la Universidad de Montreal (donde habían utilizado durante años mi monografía sobre el paralenguaje), hablé de nuestras casi ilimitadas experiencias sonoras que, consciente o inconscientemente (es decir, oyendo o también escuchando), y más allá del lenguaje humano, nos rodean y envuelven en nuestras interacciones personales y con el entorno audible, y su íntima relación con la producción de sonidos, tanto vocales como ambientales o de instrumentos musicales.

34 «Many times she allowed the dark to fall upon them, refraining from lighting the lamp. The dark discreet room, their isolation, the music that still vibrated in their ears united them».

35 «The whole empty world seemed haunted. Rustlings of the sage, seepings of the sand, gusts of the wind, the night, the loneliness».

36 «Inside it was dark, a prolonged puff of hot air, thick with the mingled odours of flowers, perfume, upholstery, and gas, enveloped her upon the instant. It was the unmistakable aroma of the theater». 
paseo [...] hablaron de pinturas y de música [...] Pero los colores de los setos [...] el gorjeo de los pinzones [...], el perfume de las malas hierbas que ya estaban quemando, el giro de su cuello, la fascinación de aquellos ojos oscuros que se posaban en él de vez en cuando, la atracción de toda su figura, le causaron una impresión más profunda que las palabras que intercambiaban. Inconscientemente, se puso más erguido, caminando con un paso más elástico» (Galsworthy, IC, $1, \mathrm{XIII})^{37}$.

Pero, además, no olvidemos que los efectos lumínicos, no solo influyen en nuestra percepción de otras personas (su voz, sus gestos) y de cuanto nos rodea, sino en nuestro propio comportamiento verbal-no verbal:

El grupo [...] alrededor de la mesa de té. Las lámparas y el fuego entrecruzaban sus destellos en la vajilla de plata y en la porcelana, en la luminosa neblina del cabello de Eiffe y en la blancura de la frente de Anna al inclinarse hacia atrás en su silla, detrás de la tetera (Wharton, $R, \mathrm{XVI})^{38}$.

¡Vivamos cada detalle!

\section{FUNCIÓN NEUTRA Y FUNCIÓN EFECTIVA DEL DISCURSO Y OTROS COMPONENTES EN UNA INTERACCIÓN}

Los componentes de una interacción cumplen dos tipos de funciones:

- una función neutra (que pudiera no serlo):

una voz pastosa de embriaguez [...] La puerta se cerró con un portazo. / Escucharon. / [...] Oyeron pasos de alguien dando bandazos escaleras arriba en la casa de al lado. Luego un portazo (Woolf, $Y$, "1910")

- una función efectiva, que afecta verdaderamente a nuestra interacción consciente o inconscientemente (ej., un portazo, el repicar de la lluvia en los cristales, pasos que se acercan, la invasión olfatoria de olores de cocina ajenos):

37 «they talked of pictures and music [...] But to Jolyon the colours of the hedges [...] the twittering of chaffinches [...], the perfume of weeds being already burned, the turn of her neck, the fascination of those dark eyes bent on him now and then, the lure of her whole figure, made a deeper impression than the remarks they exchanged. Unconsciously he held himself straighter, walked with a more elastic step».

38 «The group [...] about the tea-table. The lamps and the fire crossed their gleams on silver and porcelain, on the bright haze of Eiffe's hair and on the whiteness of Anna's forehead, as she leaned back in her chair behind the tea-urn».

39 «A thick drunken voice [...] Then the door slammed./ They listened./ [...] They could hear heavy footsteps lurching up the stairs in the next house. Then the door slammed». 
el deprimente llanto de niños por la noche, golpes secos de pasos [...] gritos roncos $[\ldots]$ el traqueteo de ruedas en el empedrado [...] los chillidos del niño y los rugidos de la madre (Crane, MGS, III) ${ }^{40}$.

\section{CANALES SENSORIALES, VISIÓN, SINESTESIA Y CRONÉMICA}

Además de la percepción sensorial (Poyatos, 1994a, cap. 2; más extensamente en 2002a, cap. 2) e intelectual de los componentes interactivos, hay tres dimensiones básicas.

- La percepción visual de los componentes de la interacción, sobre todo con la visión macular $\left(12^{\circ}-15^{\circ}\right.$ en el plano horizontal y $3^{\circ}$ en el vertical) para explorar la cara de alguien, y la visión periférica $\left(90^{\circ}\right.$ a cada lado del plano sagital: $180^{\circ}$, y $150^{\circ}$ verticalmente) para simplemente ser conscientes de la presencia de otras personas (probablemente oyendo lo que dicen o interpretando su gesticulación) y del entono sin tener que mirar, pero con una posible función en nuestra interacción:

Su rostro atento [sir Percival] se relajó un poco. Pero vi que uno de sus pies, suavemente, silenciosamente, golpeaba sin cesar la alfombra por debajo de la mesa, y tuve la impresión de que tenía, secretamente, más ansiedad que nunca (Collins, WW, 190) ${ }^{41}$.

- $\quad$ el fenómeno de la sinestesia al percibir a la gente y el entorno (Poyatos, 1994a: cap. 2; más extensamente en 2002a: cap. 2; 2009: 200202), como ocurre, visualmente, con la suavidad de un tapizado en contraste con la aspereza general sugerida por la persona rústica, y en otras asociaciones:

Oyóse asimismo un espantoso ruido, al modo de aquel que se causa de las ruedas macizas que suelen traer los carros de bueyes, de cuyo chirrío áspero y continuado se dice que huyen los lobos y los osos (Cervantes, $Q, 2$, XXXIV).

- $\quad$ La dimensión cronémica ${ }^{42}$ de la percepción auditiva, visual, táctil y cinestésica (parte de un proceso de intelectualización), puesto que

40 «the dismal wailing of babies at night, the thumping of feet [...] hoarse shoutings $[\ldots]$ the rattling of wheels on the cobbles [...] the screams of the child and the roars of the mother».

41 «His attentive face relaxed a little. But I saw one of his feet, softly, quietly, incessantly beating on the carpet under the table, and I felt that he was secretly as anxious as ever».

42 Acuñé el término chronemics/cronémica en un temprano artículo para Linguistics (Poyatos, 1972b: 84), definiéndolo y aplicándolo después ampliamente (Poyatos, 1994a: 159160; 1994b: 279; 2002a: 267-268, 284, 312; 2008c: 145-153 [en teatro y cine]). 
una palabra, una mirada, un intencionado portazo o un silencio, según su intensidad y relevancia, permanece conscientemente o no en nuestra mente, mientras que otros componentes están todavía operando en el continuo de la interacción entre emisión y recepción:

"Justo lo que supuse,” dijo Tom. "¡Y es natural!” y, con gran satisfacción, tomó un largo sorbo de su copa" (Dickens, $M C$, VI) ${ }^{43}$.

\section{LA EVALUACIÓN INTELECTUAL DEL DISCURSO Y OTROS SIGNOS SENSIBLES}

Los signos sensorialmente percibidos están sujetos a una evaluación intelectual que tiene implicaciones lingüísticas y sociopsicológicas en el discurso de los cointeractores. Utilizando el ejemplo de una interacción hombre-mujer (que mis alumnos siempre imaginaban mejor que nada), simultáneamente a la percepción mutua de sus respectivos signos sensibles (palabras, tono de voz, gestos, posturas, perfume, vestido, figura, etc.) también están siendo -a un nivel diferente, pero paralelo y más ocultopensados y evaluados mutuamente. Es como si él, por ejemplo, estuviera diciéndole a ella con el silencioso lenguaje mental:

Me estás hablando, diciéndome [tal cosa] con una voz de [tales] características, y con esos gestos, activando esos rasgos faciales (que ya de por sí me encantan) mientras percibo, aunque no mire directamente [visión periférica], tu figura y tu postura, y mientras siento tu perfume; me gusta el sonido de tu voz mientras me hablas, me atrae la manera con que acompañas tu voz con la mano y con los ojos, y la manera de moverse esos rasgos faciales al decir eso que me estás diciendo en este instante; y veo todo ello como algo perfectamente propio de toda tú; y me doy más cuenta todavía a través de ese perfume tuyo que envuelve tu voz, tus ojos, tu cara, tus manos, todo cuanto me habla, a medida que me dices lo que me dices. Son todas esas cosas juntas lo que hace que me gustes.

Y junto a las sensaciones directas están las sinestésicas, tan importantes. Y más allá de esos componentes personales internos de esa interacción, es la mutua orientación de los dos participantes, las características del lugar (ej., en penumbra y con música, o en silencio y con la lluvia suavemente golpeando los cristales), la satisfacción fisiopsicológica después de comer y beber, así como la sensación de intimidad (aunque sea un lugar público), lo que completa esa serie de procesos semiótico-comunicativos, entre los cuales no podemos aislar la estructura lenguaje-paralenguaje-kinésica, ni cualquier otro sistema de signos que opere en el intercambio interacti-

43 «"Just what I supposed," said Tom. "Quite natural!" and, in his great satisfaction, he took a long sip out of his wine-glass». 
vo. Pues bien, insistamos: todo ello -y mucho más que no tiene cabida aquí (por tanto, sin hacer justicia a su extrema complejidad)- está presente en el discurso representado en el texto literario para quien lo lea no superficial y precipitadamente, sino en profundidad, dándole vida e indentificándose en él.

Pero a medida que ella hablaba empezó a ver que la cara de Dairy no reflejaba en nada sus palabras, que seguía mostrando el aspecto abstracto de un hombre que no está escuchando lo que se le dice. Le causó una ligera punzada descubrir que sus pensamientos pudieran vagar en un momento así; entonces, en un acceso de gozo, percibió la razón. / De algún modo indefinible se había dado cuenta, sin volver la cabeza, de que estaba inmerso en la sensación de su cercanía, absorbido en contemplar los detalles de su cara y de su vestido; y este descubrimiento hizo que las palabras se agolparan en sus labios. Sintió que hablaba con soltura, autoridad, convicción. Se dijo a sí misma: "No le importa nada lo que diga -basta con que lo diga- aunque sea estúpido le gustaré más precisamente por ello..." Sabía que cualquier inflexión de su voz, cada gesto, cada característica de su persona -sus mismos defectos, el hecho de que su frente fuera demasiado alta, que sus ojos no fueran lo bastante grandes, que sus manos, aunque finas, no fueran pequeñas, y que sus dedos no terminaran en disminución- sabía que estas deficiencias eran otros tantos canales por los que su influencia fluía hasta él, que le agradaba a pesar de ellas, precisamente por ellas $[\ldots]$ y por primera vez sintió en sus venas la seguridad e ingravidez del amor feliz (Wharton, $R$, XII) ${ }^{44}$.

\section{COMPONENTES INTERACTIVOS LIBRES Y TRABADOS, MOMENTÁNEOS Y PERMANENTES}

Los componentes interactivos son libres o trabados en la mutua relación interpersonal o con el entorno, pero también pueden estar relacionados

44 «But as she talked she began to see that Dairy's face gave back no reflection of her words, that he continued to wear the abstracted look of a man who is not listening to what is said to him. It caused her a slight pang to discover that his thoughts could wander at such a moment; then, with a flush of joy she perceived the reason. In some undefinable way she had become aware, without turning her head, that he was steeped in the sense of her nearness, absorbed in contemplating the details of her face and dress; and the discovery made the words throng to her lips. She felt herself speak with ease, authority, conviction. She said to herself: 'He doesn't care what I say -it's enough that I say it- even if it's stupid he'll like me better for it...' She knew that every inflection of her voice, every gesture, every characteristic of her person -its very defects, the fact that her forehead was too high, that her eyes were not large enough, that her hands, though slender, were not small, and that her fingers did not taper- she knew that these deficiencies were so many channels through which her influenced streamed to him; that she pleased him in spite of them, because of them [...] and for the first time she felt in her veins the security and lightness of happy love». 
con componentes inteligibles (ej., la fragancia de un perfume + estatus social) y aparecer en complejos de signos:

Volvió [...] sus ojos hacia mí con una incipiente sonrisa, la más dulce melancolía y una tímida pero aguda mirada inquisitiva que hizo que las mejillas me hormiguearan con inexpresables emociones (Brontë, TWH, LIII) ${ }^{45}$;

pero esta trabazón puede ser tan momentánea y pasajera como para pasar desapercibida:

$\mathrm{Al}$ oír la palabra padre, vi que su anterior preocupación volvía a su cara. La suavizó como antes y desapareció al instante; pero había estado allí, y había aparecido de súbito con mis palabras (Dickens, $\mathrm{BH}, \mathrm{XVII})^{46}$.

Los componentes momentáneos son de muy breve duración (ej., una palabra, un suspiro, un cruce de piernas), mientras que los permanentes abarcan todo el encuentro o buena parte de él (ej., el tipo de voz de una persona, unos labios sensuales, el masticar chicle):

Mr. George, fumando no tan plácidamente como antes, pues, desde que ha entrado Judy, ha estado, en buena medida, turbado por su fascinación [...], lo cual le obliga a mirarla (Dickens, $B H, \mathrm{XXI})^{47}$.

\section{LOS RASGOS CALIFICADORES DE LOS COMPONENTES INTERACTIVOS: POSICIÓN, INTENSIDAD, DURACIÓN}

Pero, además de los componentes de una interacción, hay tres rasgos que los califican.

- Posición de cualquier actividad o no-actividad somática o no somática entre el principio y el final del encuentro, bien estrictamente temporal (donde ocurre) o funcional (donde realmente afecta a la interacción, no necesariamente el mismo punto), esta última instantánea (ej., el notar la manga raída de alguien o su voz vacilante), de mayor duración (ej., unos ojos al borde de las lágrimas durante una conversación) o abarcando toda la interacción con una persona o con el entorno (ej., la cicatriz en la cara de alguien, la fragancia de un bosque).

45 «She [...] turned her eyes upon me with a dawning smile, most sweetly melancholy, and a look of timid though keen enquiry that made my cheeks tingle with inexpressible emotions».

46 «At the word Father, I saw his former trouble come into his face. He subdued it, as before, and it was gone in an instant; but it had been there, and it had come so swiftly upon my words».

${ }^{47}$ «Mr George, smoking not quite as placidly as before, for since the entrance of Judy he has been in some measure disturbed by a fascination [...] which obliges him to look at her». 
- Intensidad, o grado de ocurrencia de las principales características de un componente (ej., algo dicho con articulación tensa, un sonrojo profundo, la duración de una postura, un maquillaje excesivo, llamando a la puerta con suavidad):

“¿Por qué tienes tanta prisa en dejarme, Helen?”-dijo [Mr. Huntingdon] con una sonrisa de la más provocativa autosuficiencia-, "tú no me odias, ¿sabes?" (Brontë, TWH, XVIII) ${ }^{48}$,

quizá con un efecto duradero que afecta a las conductas de los demás y determina su duración efectiva.

- Duración, su dimensión temporal exacta, que puede afectar a conductas periféricas y a todo el encuentro (ej., una voz de haber bebido, cuánto tiempo está sonando el teléfono), considerando el discurso de las personas y los elementos presentes (ej., mobiliario, iluminación, un perfume); y su verdadera duración efectiva, la que realmente importa, sin dejar de tener en cuenta el posible efecto intermitente (ej., un cambio en el tono muscular de la cara bajo el efecto de un insulto verbal reciente o un recuerdo recurrente).

\section{Coestructuraciones del Discurso y Otros COMPONENTES INTERACTIVOS, CON OTROS PRECEDENTES, SIMULTÁNEOS Y SIGUIENTES}

Este es el rasgo más intricado y profundo de una interacción, pues se refiere no solo a la relación de cada una de las actividades (comportamentales o no) del hablante con las otras suyas más o menos inmediatas (precedentes, simultáneas o siguientes), sino a las relaciones con las de sus cointeractores, y a las relaciones entre esos elementos interpersonales y los externos, que pueden afectarse mutuamente de diversas maneras.

- Coestructuration a posteriori, la más frecuente, como cuando el "Te quiero" de un hombre es suscitado por la mirada silenciosa de ella inmediatamente precedente (la secuencia causa-efecto, que es lo único que suele buscarse superficialmente), aunque pudo haber sido por las palabras precedentes de ella, siempre dependiendo de su posición, duración y, sobre todo, la intensidad de la conducta o no-conducta afectante, como ocurre con el silencio:

Mr. Pinch estaba en ese momento mirando pensativamente a las barras [de la chimenea]. Pero al hacer aquí su compañero una pausa [mientras

48 "Why are you in such a hurry to leave me, Helen?" -he said, with a smile of the most provoking self-sufficiency-, "you don't hate me, you know?"». 
hablaba], se sobresaltó y dijo: “;Oh! naturalmente,” y se dispuso a escuchar de nuevo (Dickens, $M C, \mathrm{VI})^{49}$.

Por otra parte, el “¡Te quiero!” de un hombre suscita en la mujer su mirada fija, pero podía haberla causado una conducta de él inmediatamente precedente, o una antes de eso, y así sucesivamente hacia atrás. Pero una conducta o no-actividad de ella inmediatamente precedente (ej., una desviada mirada lacrimosa) pudo haber servido de estímulo, o haber sido el único, para la mirada de él, como pudo haberlo sido cualquiera de las miradas anteriores de ella. Es decir, que el espacio de tiempo para la conducta presente de él podría haber sido ocupado por un componente diferente, o podría haber dicho lo que de otra forma no hubiera dicho. Es más, podría ser -dentro de la dimensión cronémica- el efecto cumulativo intermitente de las actividades o no-actividades de él o de ella lo que finalmente motiva la expresión verbal o no-verbal (incluyendo el silencio); o incluso el efecto duradero continuo de sus previas conductas o no-conductas, o ambos (ej., un mutuo abrazo anterior) actuando por encima y a través de componentes más recientes que todavía afectan en el presente, como ocurre con el impacto que tiene un grito en uno de los famosos personajes de Dickens, que observamos cómo se prolonga a través de las siguientes secuencias de actividades:

[cuando un hombre desde la ventana les grita inesperadamente a él y a sus hijas para que salgan de su propiedad] Mr. Pecksniff se puso el sombrero y caminó muy deliberadamente y en profundo silencio [efecto del grito] hacia el carruaje de alquiler, mirando con gran interés a las nubes mientras tanto [continúa el efecto]. Después de ayudar a sus hijas y a la señora Todgers a subir al vehículo, se quedó mirándolo unos minutos como si no estuviera seguro de si era un carruaje o un templo [el efecto continúa]; habiendo resuelto este punto en su mente, se metió en su sitio, extendió las manos sobre las rodillas y sonrió a las tres presentes (Dickens, $M C, \mathrm{IX})^{50}$.

A nivel del comportamiento, la duración efectiva y la posición de, por ejemplo, los rasgos faciales estáticos de una mujer, pueden haber suscitado mi mirada, o pudo haber sido su perfume o su vestido. Una actividad comportamental o no-comportamental puede haber sido provocada por

49 «Mr Pinch was just then looking thoughtfully at the bars. But on his companion pausing in this place, he started, and said "Oh! of course," and composed himself to listen again".

50 "Mr Pecksniff put on his hat, and walked with great deliberation and in profound silence to the fly, gazing at the clouds as he went, with great interest. After helping his daughters and Mrs Todgers into the conveyance, he stood looking at it for some moments, as if he were not quite certain whether it was a carriage or a temple; but, having settled this point in his mind, he got into his place, spread his hands out on his knees, and smiled upon the three beholders. / But his daughters, less tranquil-minded, burst into a torrent of indignation». 
el efecto cumulativo de, por ejemplo, el alcohol, una relación proxémica íntima o los pensamientos reales o imaginados de la otra persona; como cuando pensamos que alguien está pensando que tenemos aspecto ridículo, cuando no está pensando eso en absoluto, pero el mero hecho de que nosotros lo creamos suscita en nosotros una reacción verbal o no-verbal. Finalmente, ese efecto cumulativo y un componente momentáneo pueden a la vez suscitar una conducta, por ejemplo: una distancia interpersonal íntima + el perfume de ella + un inesperado trueno provoca un abrazo. Pero un efecto cumulativo controlable puede manipularse para suscitar la deseada conducta. Naturalmente, una coestructuración a posteriori puede resultar aún más difícil de identificar únicamente por componentes sensibles y no a la vez por los posibles efectos de actividades mentales o de asociaciones sinestésicas, perfectamente efectivos, pero muy difícil de verificar, puesto que yacen ocultos a niveles más profundos aún. Por añadidura, una reacción puede haber sido provocada por un componente muy anterior en la interacción cuando se combina con un estímulo similar al actual. Pero a veces el estímulo más importante es el de un recuerdo positivo o negativo:

"Pero tú hiciste muchas cosas desagradables a demasiadas películas que me importaban [...]." Un olvidado odio volvió a su voz. "Y lo peor es que [...]" (Mailer, DP, XVI) ${ }^{51}$.

Finalmente, la percepción de cualquier tipo de signos en un momento dado puede proporcionarnos de pronto una información especial sobre signos previos que no habíamos decodificado totalmente (o no correctamente), y este descubrimiento nos permitirá reinterpretarlos a posteriori:

Su sorprendente rapidez de percepción [Rachel] detectó un cambio en mi voz (Mr. Bruff], o en mi modo de hacerle esa pregunta, lo cual le advirtió de que yo había estado hablando todo el tiempo pensando en un objetivo ulterior. Se paró y, soltando su brazo del mío, me miró inquisitivamente a la cara (Collins, $M$, Segundo periodo, Segunda Narrativa, I) ${ }^{52}$.

- $\quad$ Coestructuración simultánea. Por ejemplo, el sonrojo de una persona puede suscitar en otra vacilación en sus palabras, paralenguaje, gestos y hasta posturas. Pero aquí hay dos niveles: la realización de, por ejemplo, lenguaje-paralenguaje-kinésica, o sonrisa-paralenguaje,

51 «But you did too many unpleasant things to too many pictures I cared about [...]." A forgotten hatred came back to his voice. "And the worst of it is [...]"».

52 «Her astonishing quickness of perception, detected a change in my voice, or my manner, when I put that question, which warned her that I had been speaking all along with some ulterior object in view. She stopped, and taking her arm out of mine, looked me searchingly in the face». 
como elementos lógicamente trabados que siempre ocurren en ciertas construcciones fijas; y el efecto realmente simultáneo de un componente sobre otro:

cuando el significado de sus palabras fue tomando forma en su mente [Anna], él [Darrow] vio aparecer un curiosa luz interior en su mirada fija (Wharton, $R, \mathrm{XX})^{53}$.

- Coestructuración a priori, finalmente, es el aspecto más ignorado de la coestructuración interpersonal y de interacción persona-entorno, debido precisamente a la atención casi exclusiva que se ha dado al modelo causa-efecto, como si algo que no ha ocurrido aún, o que puede no ocurrir nunca, no pudiera jugar una función interactiva específica en el momento presente (de hecho, puede incluir hasta componentes extrapersonales siguientes, incluso una actividad interfiriente prevista o temida):

“QQue viene!" pensó, conteniendo un poco el aliento y dejando la pluma (Woolf, $Y$, “1880") ${ }^{54}$.

- Pero la forma más interesante de esta relación a priori es la que he llamado retrocomunicación anticipada oculta (advanced hidden feedback): algo que aún no ha ocurrido afecta a la persona que lo hará y también a otra, y ciertas funciones manipulativas o positivas pueden afectar críticamente el desarrollo del encuentro:

Waythorn se sintió enrojecer anticipando la sonrisa del otro (Wharton, OT, II).

Puede ocurrir, por ejemplo, en una reunión de negocios con relación a algo que no se ha dicho aún, pero que podría ser crucial; pero también depende de sus funciones positivas, según nuestra capacidad de observación y nuestra sensibilidad:

Vio que Webley abría la boca para hablar y se apresuró a anticipar lo que imaginó que iba a ser su objeción (Huxley, $P C P, \mathrm{~V}$ ).

\section{Conclusión}

Dadas las limitaciones impuestas, se ha omitido, como ya indiqué al principio, lo que lógicamente completaría esta visión literaria de la interacción personal y con el entorno: el análisis estructural de la conversación, por lo cual solo me queda remitir a la versión más completa -del modelo

\footnotetext{
53 «as the meaning of his words shaped itself in her mind he saw a curious inner light dawn through her set look».

54 «"She's coming!" she thought with a little catch of her breath, laying down the pen».
} 
que inicié en los años setenta-y profusamente ilustrada con ejemplos literarios, en el volumen I de la trilogía de 2002 Nonverbal Communication Across Disciplines, reelaborando el de la trilogía en español La comunicación no verbal (1994). Sin embargo, por su importancia para el estudio del discurso, quisiera dar al menos la definición de conversación que siempre propuse, porque creo que sugerirá su aplicación para el análisis del discurso dentro de los temas y perspectivas tratados:

una serie de intercambios verbales y no verbales espontáneos entre al menos dos participantes (que observan, pero también trasgreden, ciertas reglas, según la cultura y otros factores) en un flujo irregular de turnos de hablante y oyente, con actividades simultáneas permitidas o no y gran número de pausas acústicas y visuales, todo estas actividades como conductas positivas o negativas.

Aun así, confío en haber conseguido al menos dos objetivos muy concretos: mostrar el gran valor de las incisivas observaciones de los escritores acerca de toda actividad o no-actividad comportamental o no-comportamental, basada en la realidad, que pueda tener una función concreta en una interacción interpersonal o con el entorno; y resaltar cómo la literatura puede ayudar enormemente al investigador en psicología y en cualquiera de las ciencias sociales, además, sobre todo, de la lingüística, las filologías universitarias o la semiótica, y no solo para acompañar a la observación natural, sino incluso la experimental. 


\section{BIBLIOGRAFÍA}

Lorenzo, E. (1999): "Kinesioterapia y la kappa griega”, $A B C$, 16-8-1999.

Poyatos, F. (1972a): "Paralenguaje y kinésica del personaje novelesco: nueva perspectiva en el análisis de la narración”, Revista de Occidente, 113/114, págs. 148-170.

- (1972b): "The Communication System of the Speaker-Actor and His Culture: A Preliminary Investigation”, Linguistics, 32, págs. 64-86.

- (1976): "Nueva perspectiva de la narración a través de los repertorios extraverbales del personaje”, en S. Sanz-Villanueva y C. Barbachano (eds.), Teoría de la novela, Madrid, SGEL, págs. 353-383.

- (1977): "Forms and functions of Nonverbal Communication in the Novel: A New Perspective of the Author-Character-Reader Relationship", Semiotica 21 (3-4), págs. 295-337.

- (1981): "Forms and functions of Nonverbal Communication in the Novel: A New Perspective of the Author-Character-Reader Relationship", en A. Kendon (ed.), Nonverbal Communication, interaction, and Gesture. Approaches to Semiotics, 41, págs. 107-149.

- (1993): Paralanguage: A Linguistic and Interdisciplinary Interactive Speech and Sounds. Ámsterdam/Filadelfia, John Benjamins.

- (1994a): La comunicación no verbal, vol. I: Cultura, lenguaje y conversación, Madrid, Istmo.

— (1994b): La comunicación no verbal, vol. II: Paralenguaje, kinésica e interacción, Madrid, Istmo.

- (1994c): La comunicación no verbal, vol. III: Nuevas perspectivas en novela y teatro y en su traducción, Madrid, Istmo.

- (1997): "El acto de lectura: su realidad verbal-no verbal”, El Extramundi y los Papeles de Iria Flavia, XI, otoño, págs. 11-60.

- (1998): "Los silencios en el discurso vivo y en la literatura: para el estudio realista del lenguaje y su entorno", Oralia, 1, págs. 47-70.

- (2001): "Escritor, personaje, lector, espectador, entorno: procesos semióticocomunicativos en teatro y cine", El Extramundi y los Papeles de Iria Flavia, XXVII, otoño, págs. 9-52.

- (2002a): Nonverbal Communication Across Disciplines, vol. I: Culture, Sensory Interaction, Speech, Conversation, Ámsterdam/Filadelfia, John Benjamins.

- (2002b): Nonverbal Communication Across Disciplines, vol. II: Paralanguage, Kinesics, Silence, Personal and Environmental Interaction, Ámsterdam/Filadelfia, John Benjamins.

- (2002c): Nonverbal Communication Across Disciplines, vol. III: Narrative Literature, Theater, Cinema, Translation, Ámsterdam/Filadelfia, John Benjamins. 
- (2002d): "Anthropology/Psychology/Sociology", en P. Gossin (ed.), Encyclopedia of Literature and Sciences, Westport/Londres, Greenwood Press, págs. 13-20.

- (2002e): "The nature, morphology and functions of gestures, manners and postures as documented by creative literature”, Gesture, XX, 1, págs. 99-117.

- (2008): Textual Translation and Live Translation: The Total Experience of Nonverbal Communication in Literature, Theater and Cinema, Ámsterdam/Filadelfia, John Benjamins.

- (2009): "Leyendo a Juan Ramón Jiménez a la luz de los estudios de comunicación no verbal", en M. V. Galloso Camacho (ed.), Estudios andaluces de lengua y literatura, Editorial GEU, págs. 193-218.

- (2012): "Realidad y problemas del discurso verbal-no verbal en interpretación simultánea o consecutiva”, Oralia, 15, págs. 279-303.

- (2013): "La Comunicación No Verbal como asignatura en Filologías Clásicas y Modernas", Didáctica. Lengua y Literatura, vol. 25, págs. 231-257.

- (2015): "Nonverbal Communication in Interaction: Psychology and Literature”, en A. Kostik y D. Chadee (eds.), The Social Psychology of Nonverbal Communication, Londres, Palgrave MacMillan, págs. 248-271. 


\section{BIBLIOGRAFÍA LITERARIA}

Agee, J. (1938): A Death in the Family, Nueva York, Avon Books, 1963. DF

Balzac, H. de (1847): La cousin Pons, París, Le livre de Poche, 1973. CP

Bhattacharya, B. (1955): He Who Rides a Tiger, Nueva Delhi, Hind Pocket Books. HHRT

Brontë, A. (1848): The Tenant of Wildfell Hall, Londres, Penguin Books, 1979. TWF

Caballero Bonald, J. (1967): Dos días de setiembre, Barcelona, Seix Barral, 1967. DDS

Cather, W. (1918): My Ántonia, Boston, Houghton Mifflin, 1954. MA

CELA, C. J. (1947): Viaje a La Alcarria, Boston: D.C, Heath, 1962. VA

Cervantes, M. de (1605-1615): Don Quijote de la Mancha (ed. M. de Riquer), Barcelona, Editorial Juventud, 1965. Q

Collins, W. (1860): The Woman in White, Londres, Penguin Books, 1974. WW

- (1868): The Moonstone, Londres, Penguin Books, 1986.

Crane, S. (1893): "Maggie: A Girl of the Streets", en Stephen Crane: Stories and Tales (R. W. Stallman, ed.), Nueva York, Vintage Books, 1959. MGS

Dickens, C. (1843-1844): Martin Chuzzlewit (illus. by Hablot K. Browne, 'Phiz') Hardsmondsworth, Penguin, 1968.

- (1849-1850): David Copperfield, Nueva York, Modern Library, 1950. DC

- (1853): Bleak House (illus. by Hablot K. Browne, 'Phiz'). Londres, Penguin Books, 1985. BH

Doctorow, E. L. (1985): World's Fair, Nueva York, Fawcett Crest. WF

Dos Passos, J. (1936): The Big Money, Nueva York, Washington Square Press, 1961. $B M$

Dreiser, T. (1900): Sister Carrie, Nueva York, Dell, 1960. SC

Eliot, G. (1859): Adam Bede, Nueva York, The Pocket Library, 1956. AB

FAulKner, W. (1931): Sanctuary, Nueva York, Signet Books, 1961. S

Galdós, B. Pérez (1881): La desheredada, Madrid, Librería de Perlado, Páez y C.a , 1909. $D$

- (1886-1887): Fortunata y Jacinta.

Galsworthy, J. (1920): In Chancery, Hertfordshire, Wordsworth Editions, 1994. IC

Grey, Z. (1909): The Last Trail, Filadelfia, The Blakiston Company, 1945. LT

- (1918): The U.P. Trail, Nueva York, Pocket Books, 1956. UPT

Hailey, A. (1963): Hotel, Nueva York, Bantam Books, 1966. H

Huxley, A. (1928): Point Counter Point, Nueva York, Avon Books. PCP

- (1936): Eyeless in Gaza, Nueva York, Bantam Books, 1961. EG

- (1914): "A Painful Case”, Dubliners. The Portable James Joyce, Nueva York, The Viking Press, 1947. $P C$

López Salinas (1960): La mina, Barcelona, Destino. $M$ 
MacLennan, H. (1945): Two Solitudes, Toronto, Macmillan of Canada, 1967. TS MaIler, N. (1955): The Deer Park, Nueva York, Signet Books, 1957. DP

Maugham, S. (1930): Cakes and Ales, Hardmondsworth, Penguin, 1960. CA

- (1903): The Pit, Nueva York, Grove Press/Londres, Evergreen Books, 1956. P

RuIz, J. Arcipreste de Hita (1283-1350): Libro de Buen Amor (ed. Alberto Blecua), Madrid, Cátedra, 1995. LBA

SÁnChez Ferlosio, R. (1958): El Jarama, Barcelona, Destino, 1958. J

Steinbeck, J. (1931): The Grapes of Wrath, Nueva York, Bantam Books, 1964. GW

STYRon, W. (1966): The confessions of Nat Turner, Nueva York, Signet Books. CNT

VALle-InCLÁn, R. M. del (1904): Sonata de primavera, Madrid, Alianza Editorial. SP West, N. (1939): The Day of the Locust, Nueva York, Bantam Books. DL

Wharton, E. (1904): "The Other Two", Great Short Works of American Realism, Nueva York, Harper \& Row, 1968. OT

- (1912): The Reef, Londres, Penguin, 1993. $R$

Wilder, T. (1938): Our Town. Acting Edition, Nueva York, Coward-McCann, 1938. $O T$

Wilson, E. (1956): Anglo-Saxon Attitudes, Nueva York, Signet. ASA

Woolf, V. (1925): Mrs. Dalloway, Hartfordshire, Wordsworth Editions, 1996. D

- (1937): The Years, Harmondsworth, Penguin, 1973. Y 